\title{
IMPORTÂNCIA DO FARMACÊUTICO RESIDENTE EM UMA UNIDADE DE TRANSPLANTE HEPÁTICO E RENAL: INTERVENÇÕES FARMACÊUTICAS REALIZADAS
}

\author{
Importance of a Resident Pharmacist in a Liver and Kidney Transplantation Unit: Pharmaceutical \\ Interventions Performed
}

\begin{abstract}
Thalita Rodrigues de Souza1, Diana Maria de Almeida Lopes², Natália Martins Freire², Geysa Andrade Salmito', Helaine Cristina Alves de Vasconcelos ${ }^{2}$, Alene Barros de Oliveira ${ }^{2}$, Alexsandra Nunes Pinheiro², Eugenie Desirèe Rabelo Néri', 2, Paula Frassinetti Castelo Branco Camurça Fernandes ${ }^{3}$, José Huygens Parente Garcia ${ }^{4}$.
\end{abstract}

\begin{abstract}
RESUMO
O papel do farmacêutico clínico no atendimento a pacientes internados tem evoluído ao longo do tempo, com ênfase no cuidado farmacêutico. Nesse cenário, o farmacêutico residente tem contribuido substancialmente, para que pacientes transplantados hepáticos e renais sejam beneficiados com as intervenções farmacêuticas, que funcionam como ferramenta efetiva para melhoria da qualidade da assistência hospitalar. Objetivo: relatar as intervenções farmacêuticas (IF) realizadas pelo farmacêutico residente nas unidade de transplante hepático e renal de um Hospital Universitário, identificando problemas relacionados com medicamentos (PRM) durante o período de internação e alta de pacientes transplantados. Métodos: estudo transversal, no qual foram analisadas intervenções farmacêuticas realizadas pelo farmacêutico residente com pacientes transplantados hepáticos e renais no período de maio a setembro de 2010. Resultados: Durante o período de estudo foram acompanhados 219 pacientes, tendo sido detectado PRM em prescrições de 47 (21,5\%) deles. Dos pacientes com PRM, 51,06\% $(n=24)$ eram do sexo feminino. Os medicamentos envolvidos nos PRM identificados foram classificados de acordo com o Anatomical Therapeutic Chemical (ATC), tendo maior prevalência aqueles relacionados ao sistema cardiovascular e nervoso, respectivamente (43,47\% e 17,39\%). Foram identificados 81 PRM, dos quais 98,75\% foram prevenidos através da IF, com aceitação de $98,76 \%$. Os médicos foram os profissionais mais contatados (97,50\%), seguido dos enfermeiros (2,50\%). Quando Classificados pelo II Consenso de Granada, os PRM de necessidade ocorreram em $68,80 \%(n=55)$, os de segurança $28,80 \%(n=23)$ e os de efetividade $2,50 \%$ ( $n=2)$. Não foi identificada diferença estatisticamente significante na aceitação da intervenção para os diferentes tipos de PRM $(p=0,873)$. Conclusões: Os resultados sugerem que a participação do farmacêutico residente na equipe assistencial é útil para prevenir a ocorrência de PRM, sendo bem aceita pelos demais membros da equipe de saúde e coopera para a segurança do processo assistencial aos pacientes transplantados hepáticos e renais.
\end{abstract}

Descritores: Transplante Hepático; Transplante renal; Cuidados Farmacêuticos.

\footnotetext{
Instituição:

1 Residência Multiprofissional em Atenção Hospitalar à Saúde. Universidade Federal do Ceará. Fortaleza, Ceará, Brasil.

2 Serviço de Farmácia. Hospital Universitário Walter Cantídio. Universidade Federal do Ceará. Fortaleza, Ceará, Brasil.

3 Serviço de Transplante Renal. Hospital Universitário Walter Cantídio. Universidade Federal do Ceará. Fortaleza, Ceará, Brasil.

4 Serviço de Transplante Hepático. Hospital Universitário Walter Cantídio. Universidade Federal do Ceará. Fortaleza, Ceará, Brasil.
}

\section{Correspondência:}

\section{Thalita Rodrigues de Souza}

Rua Cap. Francisco Pedro, 1290 - Rodolfo Teófilo - CEP 60.430-372 - Fortaleza - CE, Brasil Fone: (85) 3366.8606 / Fax: (85) 3366.8606

E-mail: thalita@huwc.ufc.br/ resmulti@huwc.ufc.br

\section{INTRODUÇÃO}

O Brasil possui atualmente um dos maiores programas públicos de transplantes de órgãos e tecidos do mundo, contando com 548 estabelecimentos de saúde e 1376 equipes médicas autorizados a realizar transplantes. O Sistema Nacional de Transplantes está presente em 25 estados do país por meio das Centrais Estaduais de Transplantes e é considerado procedimento de alta complexidade e alto custo, sendo financiado pelo Sistema único de Saúde - SUS. ${ }^{1}$

Os transplantes renais começaram a ser realizados no Brasil a partir de 1960, passando a melhorar a qualidade de vida dos pacientes portadores de doenças renais. Em 2004, foi publicada a Política Nacional de Atenção ao Portador de Doença Renal, incluindo em seu corpo tópicos específicos sobre transplantes. A insuficiência renal crônica é responsável por um crescente número de pacientes submetidos às terapias renais substitutivas, incluindo diálises e transplantes renais. Em 2007, o SUS investiu cerca de 2 bilhões de reais em diálises, procedimentos relacionados a transplantes e medicamentos. ${ }^{2}$ 
O Transplante de fígado foi realizado pela primeira vez no Brasil com sucesso em 1985 e, desde então, vem contribuindo para salvar vidas de pacientes em fase crônica progressiva e irreversível de doença hepática. Atualmente, a sobrevida do paciente portador de doença hepática crônica submetido a transplante entre o primeiro e o quinto ano varia de 70 a 90\%. Para algumas indicações, como na doença hepática crônica colestática, a sobrevida é ainda melhor. Dados epidemiológicos de transplantes hepáticos realizados no Brasil apontam tendência de crescimento, sendo também evidente a crescente participação do Nordeste no incremento desse indicador. ${ }^{3,4}$

A gravidade das doenças renais e hepáticas, seu impacto social e econômico sobre o sistema público de saúde no Brasil e a complexidade técnica no manejo dos pacientes (da fase prétransplante ao pós-transplante), requer dedicação especial por parte da equipe multidisciplinar. Em sua maioria, pacientes candidatos a transplante e aqueles no pós-transplante, apresentam várias co-morbidades e são polimedicados, tendo destaque no plano farmacoterapêutico os imunossupressores e antimicrobianos.

No esforço multidisciplinar de prover o melhor cuidado aos pacientes, o farmacêutico deve estar presente. Nesse contexto, Martin ressalta a importância e o papel clínico desempenhado pelo farmacêutico atuando nas unidades de transplantes, destacando que muitos centros incorporam o farmacêutico, face à necessidade de monitoramento dos fármacos imunossupressores e outros medicamentos utilizados pelos pacientes transplantados. ${ }^{5}$

Segundo Kaboli, os farmacêuticos egressos de cursos de especialização em Farmácia Clínica e Residência, bem como aqueles com especialização, mestrado e doutorado em farmacologia clínica podem ser incorporados às equipes de transplante e de atendimento hospitalar a pacientes críticos. Nesse cenário, vale destacar a implantação em 2010 da Residência Multiprofissional em Atenção Hospitalar à Saúde na Universidade Federal do Ceará, com área de concentração em Transplante, que forma entre outros profissionais o farmacêutico, para atuar especificamente junto a esse grupo de pacientes. ${ }^{6}$

O uso de medicamentos por pacientes transplantados hepático e renal perpassa a profilaxia, tratamento de infecções secundárias, controle de rejeição, entre outros e requer para o sucesso do transplante o monitoramento do uso, análise de incompatibilidades, manejo das interações medicamentosas clinicamente significativas, orientação sobre o uso racional dos medicamentos, acompanhamento dos níveis séricos, entre outras estratégias.

Para Rovers e Currie, a inserção do farmacêutico clínico nesse cenário, participando ativamente das visitas clínicas diárias, provendo suporte de informações à equipe médica e de enfermagem, realizando a reconciliação medicamentosa (no internamento, transferência entre unidades e alta) e atuando diretamente com o paciente e familiares, buscando aumentar a adesão e participação ativa dos pacientes na sua própria terapêutica, desponta como oportuna e relevante, sobretudo quando consideradas as possibilidades de incremento da segurança do processo assistencial. ${ }^{7}$

A atuação do farmacêutico na visita clínica diária a pacientes críticos vem sendo amplamente estudada. Leape e colaboradores, em ensaio clínico controlado, verificaram que a presença do farmacêutico na visita clínica diária a pacientes críticos foi responsável pela redução de $66 \%$ dos eventos adversos relacionados a medicamentos. ${ }^{8}$ MacLaren e colaboradores verificaram que a presença de um farmacêutico em unidade de pacientes críticos também estava relacionada à redução de custos, corroborando achados de Schumock e colaboradores que identificaram que para cada dólar investido em ações de farmácia clínica, 16,70 dólares retornavam sob a forma de benefícios ao paciente. ${ }^{9,10}$

No Brasil, a atuação clínica do farmacêutico no manejo de pacientes transplantados iniciou juntamente com os primeiros transplantes realizados no país, através da dispensação e acompanhamento ambulatorial. Nos últimos dez anos, a atuação clínica do farmacêutico junto a pacientes transplantados tem sido ampliada na assistência hospitalar, focando especialmente a segurança do uso de medicamentos, no manejo da farmacoterapia e sua monitoração. Nessas atividades, existem riscos relacionados à efetividade e segurança dos medicamentos. Dentre os riscos identificados, estão desvios de qualidade e reações adversas. A atuação do farmacêutico situa o paciente como ser ativo e co-responsável por seu tratamento. Essa atividade foi definida por Hepler e Strand, como Atenção Farmacêutica. ${ }^{11}$

Apesar de vasta literatura internacional, no Brasil ainda são incipientes estudos que avaliem o impacto da atividade clínica do farmacêutico em grupos específicos de pacientes, tais como os transplantados. ${ }^{12}$

Dentre as intervenções realizadas pelo farmacêutico clínico no ambiente hospitalar, podem ser citadas as de caráter educativo (para o cuidador, equipe multidisciplinar: médica e de enfermagem e para o paciente), revisão da terapia medicamentosa instituída, monitoramento dos resultados terapêuticos e acompanhamento desses pacientes desde o momento de seu internamento até a alta e o retorno ao ambulatório. ${ }^{7,13}$

O objetivo do presente estudo é relatar intervenções farmacêuticas realizadas durante a rotina diária do farmacêutico residente nas unidades de transplante hepático e renal de um Hospital Universitário, descrevendo os tipos de PRM identificados, bem como as intervenções realizadas durante o período de internação (da admissão até a alta) dos pacientes transplantados.

\section{MÉTODO}

\section{Desenho do estudo}

Estudo descritivo, transversal, no qual foram analisados os registros da atividade clínica de rotina do farmacêutico residente em transplante dirigida a pacientes transplantados renais e hepáticos no período de maio a setembro de 2010 em hospital universitário terciário, com 243 leitos, cujos Serviços de Transplante Hepático e Renal são referência no Norte-Nordeste para a atividade, tendo realizado em 2009147 transplantes, sendo 82 de fígado e 65 de rim. $^{14}$

A farmácia do hospital universitário está presente na assistência aos pacientes candidatos a transplante e transplantados em todas as fases. Esse atendimento inicia-se no ambulatório, por meio da dispensação e orientação quanto ao uso dos medicamentos, prossegue durante o ato 
cirúrgico com a dispensação de todos os materiais e medicamentos necessários ao transplante, perpassa o período de internação e permanece no período pós-alta com a dispensação e monitoração do uso dos medicamentos. Durante o internamento são realizados como rotina acompanhamento farmacoterapêutico, identificação de PRM, intervenções, provisão e dispensação de medicamentos, identificação e notificação de reações adversas, sendo que todas essas atividades são devidamente registradas.

A dispensação de medicamentos aos pacientes internados é realizada para os pacientes transplantados através de sistema individual para $24 \mathrm{~h}$, baseada em segundas vias de prescrições manuscritas ou digitadas.

A rotina do farmacêutico residente iniciava com o preenchimento da ficha de seguimento farmacoterapêutico do paciente. Essa ficha continha informações sobre o estado de saúde do paciente, co-morbidades, medicamentos utilizados no momento do internamento, história de alergia a medicamentos, entre outros. Em seguida, eram realizados a análise da segunda via da prescrição médica e o aviamento e conferência da dispensação dos medicamentos. Na etapa de análise da prescrição eram identificados os problemas potenciais em prescrições, Problemas Relacionados a Medicamentos (PRM), e intervenções específicas para cada questão. A identificação dos problemas era seguida da análise dos registros no prontuário do paciente, sobretudo da evolução e das informações sobre a administração de medicamentos. Após analisar as informações coletadas, eram realizadas as intervenções farmacêuticas (IF) junto ao corpo médico e de enfermagem dos Serviços de Transplante hepático e renal, sendo registradas as aceitações.

\section{Classificação dos problemas relacionados com medicamentos}

Os PRM são conceituados por Santos e colaboradores como "problemas de saúde, entendidos como resultados clínicos negativos, devidos à farmacoterapia que, provocados por diversas causas, conduzem ao não alcance do objetivo terapêtico ou ao aparecimento de efeitos não desejados". A classificação dos PRM neste estudo seguiu o $2^{\circ}$ Consenso de Granada (1999), sendo classificados em necessidade (PRM 1 - O doente tem um problema de saúde por não utilizar o medicamento que necessita; PRM 2-O doente tem um problema de saúde por utilizar um medicamento que não necessita); efetividade (PRM 3 - O doente tem um problema de saúde por uma inefetividade não-quantitativa do medicamento; PRM 4 - O doente tem um problema de saúde por uma inefetividade quantitativa do medicamento) e segurança (PRM 5-O doente tem um problema de saúde por uma insegurança não-quantitativa de um medicamento; PRM 6 - O doente tem um problema de saúde por uma insegurança quantitativa de um medicamento). ${ }^{15}$

\section{Categorização dos medicamentos}

Os medicamentos envolvidos em PRM foram categorizados de acordo com a classificação ATC (Anatomic, Therapeutic, Chemical Classification), utilizada pela Organização Mundial de Saúde. ${ }^{16}$

\section{Análise estatística}

A análise estatística foi realizada utilizando-se o programa Statistical Package for Social Science for Windows (SPSS) 16 (2007). Os valores foram expressos em frequências relativas e absolutas e medidas de tendência central. Para a análise de proporções utilizou-se testes nãoparamétricos (c2, Fisher-Freeman-Halton). O grau de significância estatística considerado foi de $\mathrm{p}<0,05$.

\section{RESULTADOS}

No período analisado, foram acompanhados 219 pacientes, tendo sido detectado PRM em prescrições de 47 (21,50\%) pacientes. Desses, 51,06\% $(n=24)$ eram do sexo feminino. Nas prescrições analisadas foram identificados 81 PRM, envolvendo 25 medicamentos diferentes.

Os medicamentos mais prevalentes envolvidos nos PRM, foram: cloridrato de propranolol, losartana potássica, nifedipina, clonidina, atenolol, maleato de enalapril, atorvastatina, bromazepam e levodopa+carbidopa. Utilizando-se a classificação ATC, as classes mais envolvidas nos PRM foram o sistema cardiovascular (43,47\%; $n=10)$ e o sistema nervoso $(17,39 \% ; n=4)$ (Figura 1$)$.

Figura 1: Classificação ATC dos medicamentos identificados nos PRM e objeto das intervenções realizadas por farmacêutico residente em serviços de transplante hepático e renal em hospital universitário, no período de maio a setembro de 2010. (Anexado em formato TIF com resolução de 96 dpi).

\section{CLASSICAÇÃO ANATÔMICO TERAPÊUTICO (ATC)}

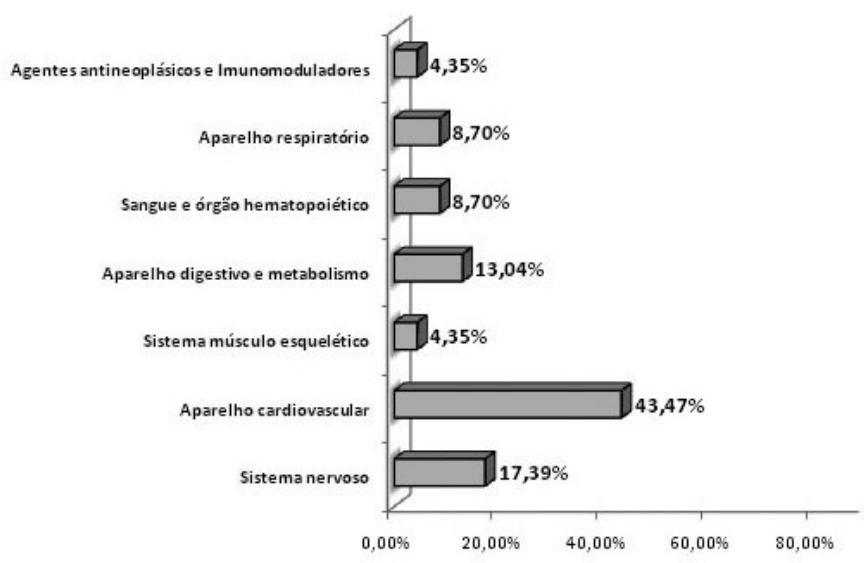

Para os 81 PRM identificados no período analisado, o farmacêutico residente realizou $81 \mathrm{IF}$, tendo sido aceitas 80 (98,76\%), conforme demonstrado na Tabela 1. A maior parte dos PRM identificados foi referente à necessidade. Não foi identificada diferença estatisticamente significante na aceitação da intervenção entre os diferentes tipos de PRM $(p=0,873)$. 
Tabela 1: Categorização dos problemas relacionados com medicamentos (PRM) potenciais, detectados por farmacêutico residente em unidades de transplante em hospital universitário, no período de maio a setembro de 2010.

\begin{tabular}{|c|c|c|c|c|c|c|}
\hline \multirow{2}{*}{$\begin{array}{c}\text { Classificação } \\
\text { dos PRM }\end{array}$} & \multicolumn{2}{|c|}{$\begin{array}{c}\text { Intervenções } \\
\text { Aceitas }\end{array}$} & \multicolumn{2}{|c|}{$\begin{array}{c}\text { Intervenções } \\
\text { não aceitas }\end{array}$} & \multirow{2}{*}{ Total } & \multirow{2}{*}{$p$} \\
\cline { 2 - 5 } & $n^{\circ}$ & $\%$ & $n^{\circ}$ & $\%$ & & \\
\hline Necessidade & 55 & $68,8 \%$ & 0 & $0 \%$ & 55 & 0,873 \\
\hline Efetividade & 2 & $2,5 \%$ & 0 & $0 \%$ & 2 & - \\
\hline Segurança & 23 & $28,8 \%$ & 1 & $100 \%$ & 24 & - \\
\hline
\end{tabular}

1. Método de classificação: Segundo consenso de Granada sobre problemas relacionados com medicamentos,1999; 2. Teste chi quadrado de person ou Teste exato de Fisher's, (significância $p=<0,05$ ).

Os PRM de necessidade identificados perfizeram um total de $68,8 \%$, tais como: falta de medicamentos, seja por prescrição de medicamento não padronizado na instituição hospitalar $(n=15)$ ou por desabastecimento da Farmácia ( $\mathrm{n}=40)$. Já os PRM de efetividade $2,5 \%$ relacionaram-se à subdoses $(n=2)$ dos medicamentos prescritos. Os PRM de segurança 28,8\% relacionaram-se à prescrições redigidas de forma inapropriada, tais como: diluições de injetáveis de modo inadequado, informação inadequada relativa à posologia, dose, forma farmacêutica, via, intervalo entre doses ou de reações adversas $(n=23)$.

Das 80 IF aceitas, 97,50\% $(\mathrm{n}=78)$ foram dirigidas aos médicos e $2,50 \%(n=2)$ aos enfermeiros (Figura 2). Não foi identificada diferença estatisticamente significante na aceitação por parte do profissional contatado quanto aos tipos de PRM $(p=0,300)$.

Figura 2: Profissionais contactados durante as intervenções realizadas por farmacêutico residente, em hospital universitário, no período de maio a setembro de 2010

\section{PROFISSIONAIS CONTACTADOS NAS INTERVENÇÕES} FARMACEUUTICAS

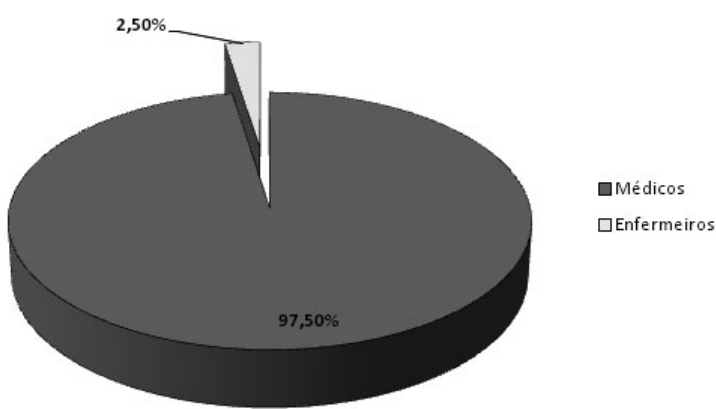

\section{DISCUSSÃO}

A intervenção farmacêutica é o momento no qual o farmacêutico, em contato com o médico ou enfermeiro realiza recomendações e apresenta evidências para as mesmas, dando suporte aos demais profissionais para escolha e adoção das melhores práticas na assistência ao paciente, sobretudo no tocante à farmacoterapia, incluindo a avaliação do uso dos medicamentos e impacto sobre a qualidade de vida. ${ }^{17}$ A IF pode ser resultante da identificação de PRM, devendo ser contínua e incorporada ao cotidiano da farmácia hospitalar. ${ }^{7}$

Os problemas durante as várias fases da assistência hospitalar à saúde (prescrição, dispensação e administração de medicamentos) estão geralmente associados a maiores riscos aos pacientes. Admitir a existência de problemas, que em sua grande maioria estão associados à condição humana, é o primeiro passo para evitá-los e tornar a assistência mais segura. O Institute of Medicine dos Estados Unidos da América aborda amplamente desde 1999 esses problemas e as formas de evitá-los, estando nelas inserida a presença do farmacêutico na análise da farmacoterapia e realização de intervenções farmacêuticas. ${ }^{7}$

A implementação da IF como atividade rotineira no atendimento a pacientes transplantados é uma das ações para reduzir a chance de que as falhas do processo assistencial relacionadas à farmacoterapia atinjam o paciente e demonstra compromisso com a qualidade da assistência..$^{7-9}$

No presente estudo, foi possível verificar que cerca de 1/5 dos pacientes internados nas unidades de transplante hepático e renal necessitaram de IF com relação às suas prescrições, tendo estas ampla aceitação por parte dos demais membros da equipe de saúde.

A aceitação das IF obtida no presente estudo $(98,76 \%)$ foi maior do que aquela obtida nos estudos de Nunes e colaboradores $(70,0 \%)$ e de Alano (79,0\%), podendo ser reflexo da boa integração entre o farmacêutico e os demais membros da equipe, além da preocupação constante com a qualidade e segurança da assistência prestada. ${ }^{18,19}$

A integração e boa comunicação entre os membros da equipe assistencial é fator contribuinte para a segurança do paciente. ${ }^{8,9}$

Os medicamentos mais envolvidos nos PRM identificados pertenciam ao sistema cardiovascular, sendo indicativo da necessidade de maior atenção por parte da equipe multiprofissional sobre tais medicamentos no tocante à prescrição, dispensação e administração. Dados semelhantes foram identificados por Nunes e colaboradores (31,72\% - sistema cardiovascular e $15,54 \%$ - sistema nervoso) e Alano (30,2\% - sistema cardiovascular e $27,5 \%$ - sistema nervoso). ${ }^{18,19}$

Os PRM de necessidade corresponderam à maior parte dos problemas identificados. Com a participação direta do farmacêutico na monitoração da farmacoterapia, a prescrição de medicamentos não padronizados e, portanto, não disponíveis na instituição, era prontamente agilizada pelo farmacêutico residente junto ao setor responsável pela aquisição, mantendo a equipe permanentemente informada e as condutas alternativas sugeridas até a chegada do medicamento. Diante do problema da ruptura do estoque, o farmacêutico residente atuava através da identificação e sugestão de alternativas terapêuticas disponíveis, e funcionando como elo entre a estrutura logística da instituição para a solução definitiva do problema. O contato entre o farmacêutico residente e os responsáveis pela logística de suprimentos da instituição foi 
realizado a cada problema identificado, facilitando sua correção, eliminando reflexos negativos para pacientes e processo assistencial.

Os casos de PRM de efetividade e de segurança estavam principalmente associados a problemas de redação das prescrições, os quais poderiam ser sanados através da informatização das prescrições, e que foram identificados e corrigidos antes da dispensação e administração do medicamento, portanto sem reflexos negativos no processo assistencial. Problemas semelhantes foram identificados em outros hospitais universitários brasileiros por Cruciol-Souza e colaboradores, Sebastião e Louro e colaboradores ${ }^{20-23}$

Os dados deste estudo dão indicações de que a informatização do processo de prescrição e a educação continuada da equipe de saúde para a prescrição, dispensação e administração racional de medicamentos podem ser medidas que, se adotadas, colaborarão para a manutenção de um ambiente assistencial mais seguro. A implementação das ações sugeridas para a segurança do processo assistencial reveste-se de especial importância quando consideramos que o estudo foi conduzido em um hospital universitário.

\section{CONCLUSÃO}

Os resultados obtidos no presente estudo sugerem que a participação do farmacêutico residente na equipe assistencial de transplante hepático e renal do hospital universitário é importante, necessária e amplamente aceita pela equipe médica e de enfermagem, contribuindo para o contínuo aprimoramento da qualidade da assistência prestada aos pacientes.

\section{ABSTRACT}

The role of clinical pharmacists in the patient care in hospitals has evolved over time, with emphasis on pharmaceutical care. In this scenario, the resident pharmacist has substantially contributed to liver and kidney transplant patients and to the benefits from pharmaceutical interventions that act as an effective tool to improve the quality of the hospital care. Purpose: To report pharmaceutical interventions (PI) performed by the resident pharmacist in a kidney and liver transplantation unit at a University Hospital, identifying drug related problems (DRP) along the admission period and discharge of transplanted patients. Methods: A retrospective study analyzing pharmaceutical interventions made by the resident pharmacist in kidney and liver transplanted patients from May to September, 2010. RESULTS: During the studied period, 219 patients were followed, and among them, it was detected 47 DPR (21.50\%). From those patients, 51.06\% $(\mathrm{n}=24)$ were female. Drugs involved in the identified DPR were classified according to the Anatomical Therapeutic Chemical (ATC), with a higher prevalence of the cardiovascular and nervous systems, $43.47 \%$ and $17.39 \%$ respectively. It was identified $81 \mathrm{DPR}, 98.75 \%$ of them were prevented through the PI, with $98.76 \%$ acceptance. Doctors were the most requested professional $(97.50 \%)$, followed by nurses $(2.50 \%)$. When ranked by the II Granada Consensus, needing DPR occurred in $68.80 \%(\mathrm{n}=55)$, safety: $28.80 \%$ ( $\mathrm{n}=23$ ), and effectiveness, $2.50 \%$ $(n=2)$. It was found no statistical significance in the intervention acceptance among the different DPR $(p=0.873)$. Conclusions: Results suggest that the participation of a resident pharmacist in the medical care is useful to prevent DRP, and it is well accepted by other team members, contributing for a safe and necessary treatment for liver and kidney transplanted patients.

Keywords: Liver Transplantation; Kidney Transplantation; Pharmaceutical Care.

\section{REFERÊNCIAS}

1. Brasil, Ministério da Saúde. Disponível em:<<http://http://portal.saude.gov.br/portal/ saude/area.cfm?id_area=1004 >>, Acesso em 01/10/10.

2. Guerra AAJ, Acúrio FA, Andrade EIG, Cherchiglia ML, Comini MC, Queiroz COV, Silva GD. Ciclosporina versus tacrolimus no transplante renal no Brasil: uma comparação de custos. Cad. Saúde Pública. 2010;26:163-74.

3. Tomé S, Well JT, Said A, Lucery MR. Quality of life after liver transplantation. A systematic review. J. Hepatology. 2008;48:567-77.

4. Portela MP, Néri EDR, Fonteles MMF, Garcia JHP, Fernandes MEP. . O custo do transplante hepático em um hospital universitário do Brasil. Rev. Assoc. Med. Bras. 2010;56:322-26

5. Martin JE, Zavala EY. The expanding role of the transplant pharmacist in the multidisciplinary practice of transplantation practice of transplantation. Clinical Transplantation. 2004;18:50-54.

6. Kaboli PJ, Hoth AB, McClimon BJ. Clinical Pharmacists and Inpatient Medical Care. A Systematic review. Arch. Intern. Med. 2006;166:955-63.
7. Rovers, JP, Currie, JD. Guia Prático da Atenção Farmacêutica. Pharmabooks. 3a edição. 2010. p.305.

8. Leape et al. Pharmacist participation on physician rounds and adverse drug events in the intensive care unit. JAMA. 1999;282:267-70 (18) (level II evidence).

9. MacLaren et al. Clinical and economic outcomes of involving pharmacists in the direct care of critically ill patients with infections. Crit Care Med. 2008 Vol. 36, No. 12.

10. Schumock GT, Meek PD, Ploetz PA, et al: Economic evaluations of clinical pharmacy services-1988-1995. The Publications Committee of the American College of Clinical Pharmacy. Pharmacotherapy 1996;16:1188-208.

11. Hepler CD, Strand LM. Opportunities and Responsibities in Pharmaceutical Care. Am. J. Hosp. Pharm. 1990;47:533-43.

12. Romano-Lieber NS, Teixeira JJV, Farhat FCLG, Ribeiro E, Crozatti MTL, Oliveira GSA. Revisão dos estudos de intervenção do farmacêutico no uso de medicamentos por pacientes idosos. Cad. Saúde Pública. 2002;18:1499-507. 
13. Klein A, Otto G, Kr"mer. Impact of a Pharmaceutical Care Program on Liver Transplant Patients' Compliance with Immunosuppressive Medication: A Prospective, Randomized, Controlled Trial Using Electronic Monitoring. Transplantation. 2009;87:839-47.

14. Hospital Universitário Walter Cantídio. Histórico de Fundação da Instituição. [acessado em 22 de setembro de 2010] Disponível em: http//www.huwc.ufc.br/ instituição/histórico.

15. Santos H, Iglésias P, Llimós-Férnández F, Faus MJ, Rodrigues LM. Segundo consenso de Granada sobre Problemas Relacionados com medicamentos: Acta médica portuguesa. 2004;17:56-66.

16. World Health Organization Collaborating Centre for Drug Statistics Methodology (WHOCC). Anatomical Therapeutic Chemical Classification (ATC code) 2010 [acesso em: 22 agost. 2010]. Disponível em: http://www.whocc.no/atc_ddd_index/.

17. Amaral MFZ, Amaral RG, Provin MP. Intervenção Farmacêutica no Processo de cuidado farmacêutico: Uma revisão. Revista eletrônica de farmácia. 2008;1:60-6.

18. Alano GM, Galato D. Indicadores do Serviço do Serviço de Atenção Farmacêutica
(SAF) da Universidade do Rio Grande do Sul de Santa Catarina. Revista de Ciência \&Saúde Pública. 2010;461-8.

19. Nunes PCH, Pereira BMG, Nominato JCS, Albuquerque EM, Silva LFN, Castro IRS, Castilho SR. Intervenção farmacêutica e prevenção de eventos adversos. Rev. Bras. Cienc. Farm. 2008;44:691-9.

20. Gommans J, Mcintosh P, Bee S, Allan W. Imoproving the quality of written prescriptions in a general hospital: the influence of 10 years of serial audits and targeted interventions. Int Med J. 2008;38:243-8.

21. Cruciol-Souza JM, Thomson JC, Catisti DG. Avaliação de prescrições medicamentosas de um hospital universitário brasileiro. Rev Bras Educ Med. 2008;32(2):188-96.

22. Sebastião ECO. Avaliação do cumprimento das exigências legais em ordens médicas em serviço de farmácia hospitalar de Ouro Preto e implicações na qualidade assistencial ao paciente. Rev Ciênc Farm. 2002;23(1):71-85.

23. Louro E, Romano-Lieber NS, Ribeiro E. Eventos adversos a antibióticos em pacientes internados em um hospital universitário. Rev Saúde Pública 2007;41(6):1042-8. 